\title{
Setting up an acupuncture knee clinic under Practice Based Commissioning
}

\section{Jonathan Freedman and Marion Richardson}

\begin{abstract}
This paper outlines the setting up of a new service in primary care offering acupuncture to patients with severe osteoarthritis of the knee. The high volume clinic is funded under the Practice Based Commissioning initiative and is the first of its type in the UK. It would appear to offer a model for similar services elsewhere.
\end{abstract}

\section{Key words: acupuncture, OA knee, Practice Based Commissioning}

\section{Introduction}

Practice Based Commissioning (PBC) was introduced in April 2005 as a central tenet of government reforms of the National Health Service (NHS) at that time. The Audit Commission saw it as a way of managing financial risk, and of improving services and the use of resources ${ }^{1}$. Amongst other things, PBC gave an opportunity for primary care practices to manage their own budgets and to commission and redesign services, making them more appropriate and convenient for patients and more cost effective. Payment is by results and practices keep up to $70 \%$ of the savings they make to reinvest in patient services. Clinicians have seized the opportunity to become patient advocates and to redesign care pathways and, in the local area, this has led to the development of Clinical Assessment (and/or Treatment) Services [CA(T)S], including one for musculoskeletal (MSK) conditions.

The MSK CA(T)S was set up in 2006 with a local GP as the clinical lead and is the conduit for all MSK referrals. Initial resistance from local colleagues has declined over time as confidence in the system has grown and savings have been demonstrated. Funding is now through the PBC budget and there are strong links with secondary care. It has been possible to manage and redesign care pathways under the auspices of the MSK CA(T)S and this paper outlines the recent success in commissioning acupuncture for patients with severe osteoarthritis (OA) of the knee.

\section{Evidence from the literature}

Osteoarthritis of the knee is common ${ }^{2}$ and debilitating ${ }^{3}$, usually occurring in the older population where co-morbidity may make surgical intervention undesirable or impossible. It is recognised that pain relief using non-steroidal anti-inflammatory drugs can be harmful and there is lack of evidence of their long-term effectiveness ${ }^{4}$. Knee replacement surgery may give suboptimal results ${ }^{5}$, has relatively high rates of complications ${ }^{6}$ and is expensive ( $f 5633$ according to the PBC tariff 2008/9) ${ }^{7}$

The literature relating to acupuncture for OA knee has been well documented. Vas et al ${ }^{8}$ demonstrated that acupuncture was effective at reducing pain and improving function as well as reducing patients' consumption of diclofenac. Berman et $\mathrm{al}^{9}$, Witt et $\mathrm{al}^{10}$ and Scharf et al ${ }^{11}$ all demonstrated improved function and pain relief using acupuncture for the treatment of OA knee. These studies, along with others, allowed White et $\mathrm{al}^{12}$ to conclude, in their systematic review of the literature, that acupuncture is significantly superior to sham acupuncture and to 
no intervention in improving pain and function. They note that evidence to support the long term effects of acupuncture in OA knee are inconclusive at present.

In addition, acupuncture has been shown to be a safe treatment. Papers by Yamashati et al ${ }^{13}$ and White et $\mathrm{al}^{14}$ demonstrated that there is negligible risk of harm to patients when acupuncture is given by competent practitioners.

\section{Previous experience of acupuncture for OA knee}

The first author works as a GP in a large (17,000 patients) city centre practice in St Albans and has been providing an acupuncture service there for the past 15 years both in a dedicated weekly clinic and in 'ad hoc' appointments. During this time, he has successfully treated many patients with OA knee and documented an $80 \%$ response rate ${ }^{15}$. There has been a long struggle to get acupuncture recognised and funded in general practice and in 2005 a voluntary donation scheme was introduced ${ }^{16}$ to help offset the perceived costs of offering this service to patients.

This experience and the anecdotal paper by $\mathrm{Camp}^{17}$, a consultant rheumatologist who noted that acupuncture is very effective for OA knee and has a lasting benefit in $60 \%$ of patients, were powerful motivators in pushing for the formal provision of acupuncture for these patients. Further work by Williamson ${ }^{18}$ demonstrated the success of acupuncture for such patients in a randomised controlled trial in a hospital setting and this further fuelled determination. The final turning point came from work by Berkowitz et al ${ }^{19}$ who demonstrated that it was possible to provide acupuncture in a dedicated hospital clinic seeing a high volume of patients. If in a hospital setting, why not in a primary care setting?

\section{Estimates of cost savings}

It was acknowledged as important to be able to demonstrate cost savings from any new service and the limited evidence available suggested that this was possible. Lindell et al ${ }^{20}$ working in a GP setting, showed that acupuncture costs significantly less than treatment in a hospital setting and demonstrated an average saving per patient of $£ 232$. Reinhold et $\mathrm{al}^{21}$ in Germany concluded that acupuncture in addition to usual care was a cost effective treatment and UK work by Thomas et al $^{22}$ studying acupuncture for back pain showed that the incremental QALY (Quality Adjusted Life Years) compared favourably with other NHS approved treatments.

\section{Meeting the commissioning criteria}

The proposal was put forward to offer a new MSK CA(T)S service to patients with severe OA knee, providing acupuncture as an alternative to referral for knee surgery. A number of potential benefits were postulated:

- Reduce the demand for outpatient, inpatient and day case treatment and so either save the cost of those activities and/or reduce waiting times

- Reduce the need for operative interventions especially knee surgery

- Reduce referrals to the local NHS physiotherapy service and thereby reduce waiting times 
- Promote a secondary to primary shift of care in line with the aims of government, Investing in Your Health and Primary Care Trusts

- Enhance patient choice and ensure a service that is locally accessible to patients in line with the stated aims of the government and local health economy

\section{- Reduce expenditure on medication}

Strict referral criteria were regarded as essential and included:

- Patients already on maximum tolerated analgesia and still experiencing pain

- Patients experiencing waking at night due to pain

- Patients with significant reduced walking e.g. 50 metres or less

Constant and consistent requests and revised proposals were submitted over many months and eventually it was agreed that the St Albans and Harpenden MSK CA(T)S Knee Clinic would be funded from savings made by the MSK CA(T)S team. These savings needed to be identifiable against the tariff prices.

No money was given for the set up costs and time and none is available for leadership, evaluation or audit. However, the purchase of 6 electro-acupuncture machines was funded. It was agreed that the GP practices would receive $\mathrm{f} 20$ per treatment from the MSK CA(T)S savings and the service would be reviewed after six months.

One major triumph was the agreement that treatments should not be limited and that ongoing maintenance treatment was approved.

\section{The current situation}

The service has been offered to patients since January 2008 and to date 51 patients have been treated. Two sites in St Albans are taking part, both with GPs who are BMAS members. The service is nurse led at both sites (again by BMAS members) and this is clearly acceptable to patients. Referral is made by the patient's GP to the MSK CA(T)S team and patients are assessed by the triage team of the lead GP and extended scope physiotherapist. If they meet the inclusion criteria (see above), they are contacted by telephone and offered acupuncture at one of the two surgeries and sent an information leaflet outlining the service. Those patients who opt out are given appointments at the orthopaedic out patient clinic.

Once patients arrive at the surgery, a clear pathway is followed - see Table 1 
TABLE 1- KNEE ACUPUNCTURE CLINIC: TREATMENT JOURNEY

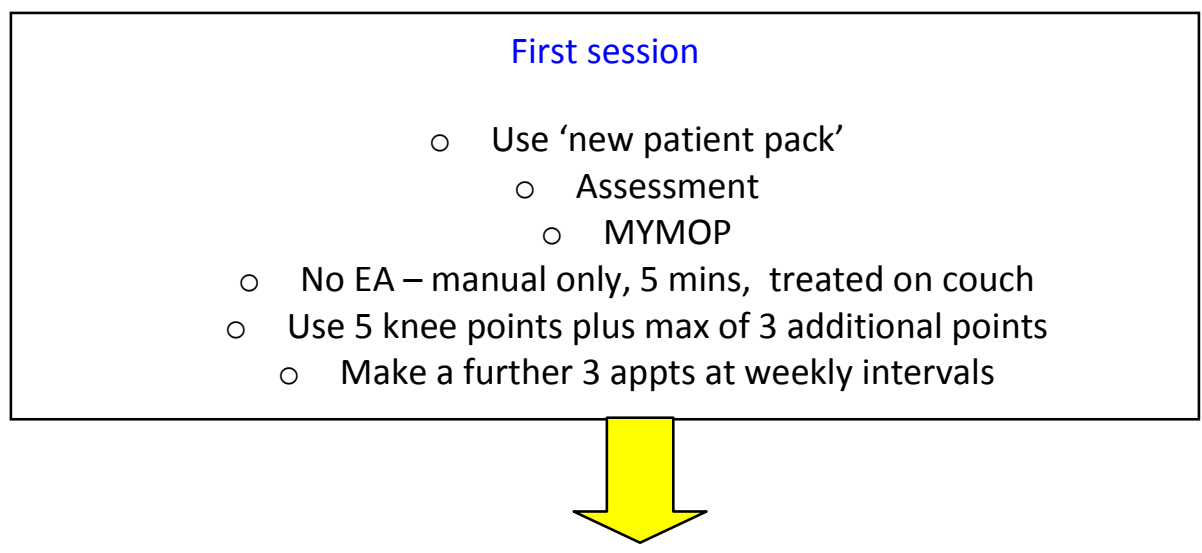

$$
\text { At } 4^{\text {th }} \text { treatment session }
$$

- Decide re further treatment

- If responding 'well' - continue manual acupuncture

- Little or no response to EA group

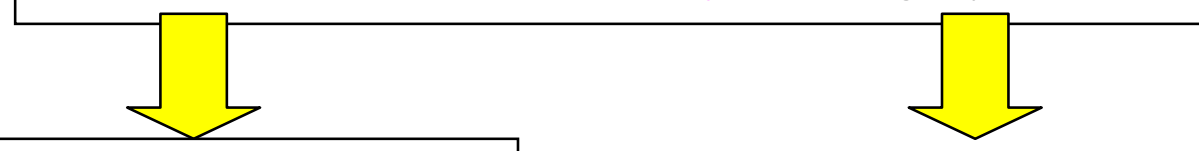

Good response group

○ Treatment in 2 weeks

o Treatment in 2 weeks

o Treatment in 3 weeks

o Treatment in 4 weeks

o Treatment in 4 weeks

o Treatment in 6 weeks

- MYMOP assessment

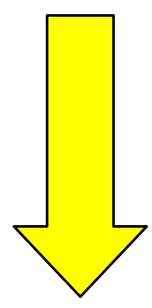

Follow-up not less than 6 weekly - about 8 treatments/ yr
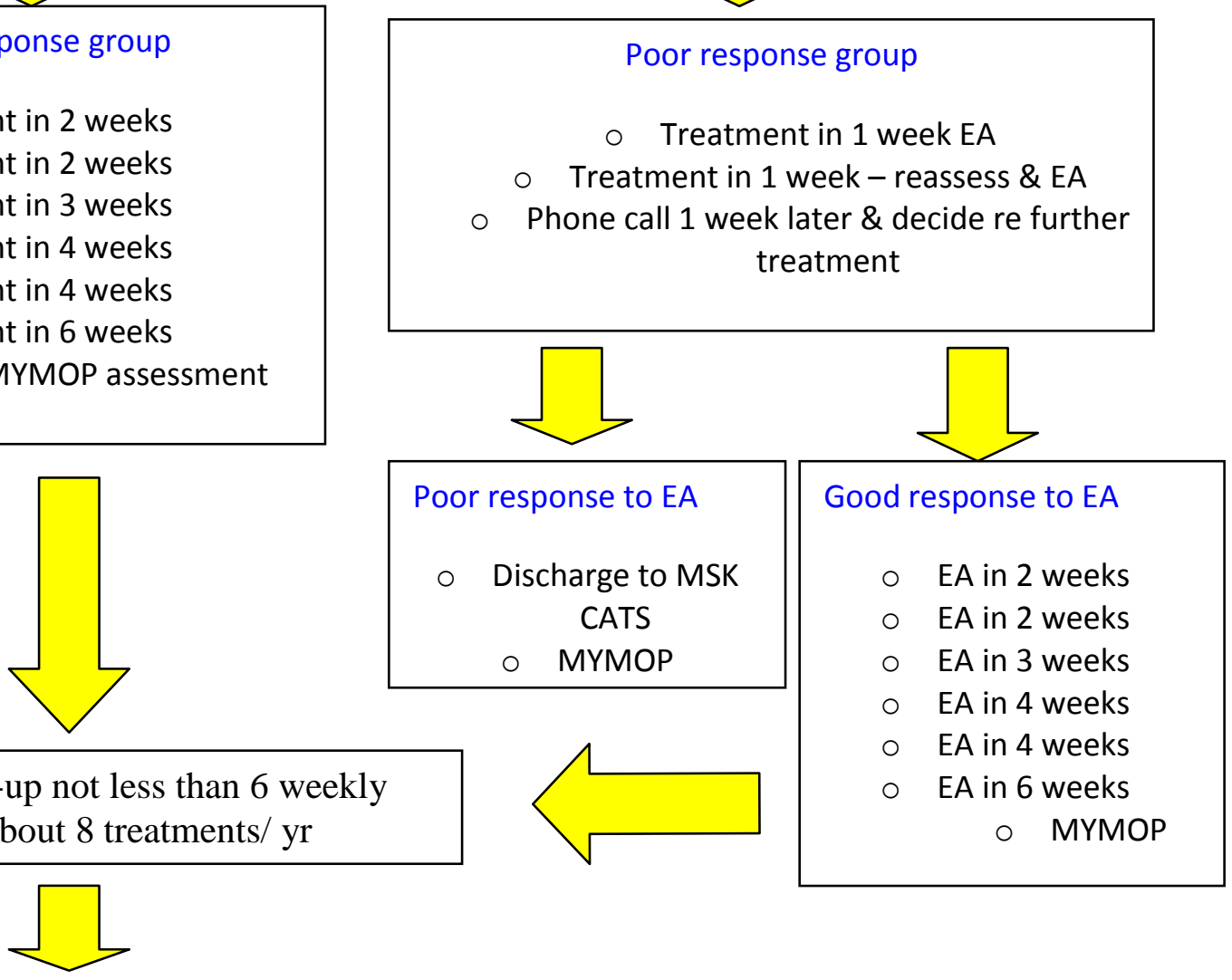

1 year

MYMOP 


\section{Discussion}

- After much consideration, a pragmatic decision was taken to use MYMOP (Measure Your own Medical Outcome Profile $)^{23}$ as the assessment tool. This tool was developed by Paterson, a GP and BMAS member and is qualitative, patient centred and easy to understand and administer. We chose to focus on pain and stiffness as the primary symptoms and accept that in doing so, we may have reduced the validity of the tool. We believe, however, that this is enabling us to measure changes in these two major symptoms of OA knee. In addition, we are able to measure changes in an activity chosen by the patient which their OA knee is making difficult, as well as their general feeling of wellbeing.

- We chose only to use electroacupuncture (EA) for those patients in whom manual acupuncture was not effective. Many of the published research studies have used EA from the outset but personal clinical experience suggested that this was not necessary for all patients and we anticipate that our evaluations will confirm this.

- To date only 6 patients have been referred back to the MSK CA(T)S team for orthopaedic referral. The remaining patients are continuing with the treatment protocol and the first few patients have now reached the maintenance phase.

- The recent 6-month review highlighted general satisfaction with the service and a continuation of the funding. This is despite the publication, since the service began, of NICE (National Institute of Clinical Excellence) guidelines ${ }^{24}$ suggesting that there 'is not enough consitent evidence of clinical or cost effectiveness to allow a firm recommendation on the use of acupuncture for the treatment of osteoarthritis'.

- Most of the referrals have been appropriate and those that have not met the inclusion criteria have been referred back to the MSK CA(T)S team before commencing acupuncture treatment.

- There are times when the 'gut feeling' of the acupuncturist has led to minor departures from the treatment protocols (the 'art' of acupuncture as opposed to the 'science'). We recognise that this would be unacceptable in a research study but feel it is valid in order to fully evaluate this new service.

- The service is free to patients, a central principle of the NHS, and this can only be achieved economically if patients are treated in small groups. All patients are told on their first visit that this will be the case. Men and women are treated separately and the group setting does seem to have a therapeutic value of its own, with patients encouraging one another and sharing their successes. Several have commented that they look forward to coming for treatment. 
- The clinics at the two surgeries will eventually reach capacity and it may be necessary to recruit a third practice. There are a number of practicing BMAS members in the area and it is anticipated that this will not pose a problem.

\section{Conclusion}

Whilst there is insufficient data at present to undertake any meaningful analysis, the initial response to the service from clinicians and patients is encouraging. Perhaps the most meaningful outcome measure in such a venture is whether the patients are happy to continue receiving acupuncture treatment rather than being referred for surgery. Using this outcome measure, we feel that we can claim success for the new service at this stage.

This PBC funded acupuncture clinic has set a precedent for such services and appears to provide a useful model which others might follow.

\section{$\underline{\text { References }}$}

1. Audit Commission. Putting commissioning into practice; implementing practice based commissioning through good financial management. London: Audit Commission, 2007.

2. Peat G, McCarney R, Croft P. Knee pain and osteoarthritis in older adults: a review of community burden and current use of primary heath care. Ann Rheum Dis (2001); 60(2) 91-7

3. Urwin $M$, Symmons $D$, Allison $T$, Brammah $T$, Busby $H$, Roxby $M$ et al. Estimating the burden of musculoskeletal disorders in the community; the comparative prevalence of symptoms at different anatomical sites, and the relation to social deprivation. Ann Rheum Dis 1998;57(11):649-655

4. Bjordal JM, Ljunggren AE, Klovning A, Slørdal L. Non-steroidal anti-inflammatory drugs, including cyclo-oxygenase-2 inhibitors, in osteoarthritic knee pain: meta-analysis of randomised placebo controlled trials. BMJ (2004); 329 (7478): 1317

5. Moseley JB, O'Malley K, Petersen NJ, Menke TJ, Brody BA, Kuykendall Dh et al. A controlled trial of arthroscopic surgery for osteoarthritis of the knee. N Engl J Med 2002; 347(2):81-88

6. Hawker GA. Who, when and why total joint replacement surgery? The patient's perspective. Osteoarthritis Curr Opin Rheumatol 2006; 18(5): 526-30)

7. http://www.dh.gov.uk/en/Managingyourorganisation/Financeandplanning/NHSFinancialRef orms/index.htm

8. Vas J, Méndez C, Perea-Milla E, Vega E, Panadero MD, León JM et al. Acupuncture as a complementary therapy to the pharmacological treatment of osteoarthritis of the knee: randomised controlled trial. BMJ (2004); 329(7476):1216-1219 
9. Berman BM, Lao L, Langenberg P, Lee WL, Gilpin AMK, Hochberg M. Effectiveness of acupuncture as adjunctive therapy in osteoarthritis of the knee - a randomised controlled trial. Ann Intern Med (2004) 141 (Dec), 901-910

10. Witt C, Brinkhaus B, Jena S, Linde K, Streng A, Wagenpfiel et al. Acupuncture in patients with osteoarthritis of the knee: a randomised trial. Lancet (2005);:366 (9480): 136-43

11. Scharf HP, Mansmann U, Streitberger K, Witte S, Kramer J, Maier $C$ et al Acupuncture and knee arthritis: a three - armed randomised trial. Ann Intern Medicine (2006) 145: 12-20

12. White $\mathrm{A}$, Foster $\mathrm{N}$, Cummings $\mathrm{M}$, Barlos PThe effectiveness of acupuncture for osteoarthritis of the knee - a systematic review. Acupuncture in Medicine (2006); 24 (5), 40-48.

13. Yamashita H, Masuyama S, Otsuki K, Tsukayma H. Safety of acupuncture for osteoarthritis of the knee - a review of randomised controlled trials, focusing on specific reactions to acupuncture Acupuncture in Medicine (2006); 24(5), 49-52

14. White A. The safety of acupuncture - evidence from the UK Acupuncture in Medicine (2006); 24 (Suppl)

15. Freedman J. An Audit of 500 Acupuncture Patients in General Practice Acupuncture in Medicine (2002); 20(1), 30-34

16. Freedman J, Richardson $M$ Introducing voluntary donations to fund primary care acupuncture - a user survey. Acupuncture in Medicine (2005); 23(3), 137-140.

17. Camp V. Acupuncture of the Knee. Acupuncture in Medicine (1992); X (2), 5764

18. Williamson L. Severe knee osteoarthritis: a randomised controlled trail of acupuncture, physiotherapy (supervised exercise) and standard management for patients awaiting knee replacement. Rheumatology (September 2007) 46:1445-1448.

19. Berkowitz S, Cummings M, Perrin C, Ito R. High volume acupuncture clinic (HVAC) for chronic knee pain. Acupuncture in Medicine (2008); 26 (1), 46- 50.

20. Lindell S. Is Acupuncture for pain relief in general practice cost effective Acupuncture in Medicine (1999); XVII (2), 97-101

21. Reihnhold T, Witt C, Jena S, Brinhaus B, Willich SN. Quality of life and cost-effectiveness of acupuncture treatment in patients with osteoarthritis pain. Eur J Health Econ (2008) 9: 209219

22. Thomas K J, MacPherson H, Ratcliffe J, Thorpe L, Brazier J, Campbell M, et al. Longer term clinical and economic benefits of offering acupuncture care to patients with chronic low back pain. Health Technol Assess (2005); Vol 9, No 32.

23. Paterson $C$ Measuring outcomes in Primary Care: a patient generated measure, MYMOP, compared with the SF-36 health survey BMJ (1996); 312:1016-1020

24. http://www.nice.org.uk/guidance/index.jsp?action=bylD\&o=11926 